\title{
Quasi-derivations and QD-algebroids
}

\author{
Janusz Grabowski* \\ Mathematical Institute, Polish Academy of Sciences \\ ul. Śniadeckich 8, P.O. Box 21, 00-956 Warszawa 10, Poland \\ e-mail: jagrab@impan.gov.pl
}

\begin{abstract}
Axioms of Lie algebroid are discussed. In particular, it is shown that a Lie QD-algebroid (i.e. a Lie algebra bracket on the $C^{\infty}(M)$-module $\mathcal{E}$ of sections of a vector bundle $E$ over a manifold $M$ which satisfies $[X, f Y]=f[X, Y]+A(X, f) Y$ for all $X, Y \in \mathcal{E}, f \in C^{\infty}(M)$, and for certain $\left.A(X, f) \in C^{\infty}(M)\right)$ is a Lie algebroid if $\operatorname{rank}(E)>1$, and is a local Lie algebra in the sense of Kirillov if $E$ is a line bundle. Under a weak condition also the skew-symmetry of the bracket is relaxed.
\end{abstract}

Key words: Lie algebroids; vector bundles; derivations; Poisson brackets.

\section{Introduction}

A Lie algebroid (or its pure algebraic counterpart - a Lie pseudoalgebra) is an object so natural that the people often use it not even realizing this fact.

Definition. Let $R$ be a commutative and unitary ring, and let $\mathcal{A}$ be a commutative $R$-algebra. A Lie pseudoalgebra over $R$ and $\mathcal{A}$ is an $\mathcal{A}$-module $\mathcal{E}$ together with a bracket $[\cdot, \cdot]: \mathcal{E} \times \mathcal{E} \rightarrow \mathcal{E}$ on the module $\mathcal{E}$, and an $\mathcal{A}$-module morphism $a: \mathcal{E} \rightarrow \operatorname{Der}(\mathcal{A})$ from $\mathcal{E}$ to the $\mathcal{A}$-module $\operatorname{Der}(\mathcal{A})$ of derivations of $\mathcal{A}$, called the anchor of $\mathcal{E}$, such that

(i) the bracket on $\mathcal{E}$ is $R$-bilinear, alternating, and satisfies the Jacobi identity:

$$
[[X, Y], Z]=[X,[Y, Z]]-[Y,[X, Z]] .
$$

(ii) For all $X, Y \in \mathcal{E}$ and all $f \in \mathcal{A}$ we have

$$
[X, f Y]=f[X, Y]+a(X)(f) Y
$$

(iii) $a([X, Y])=[a(X), a(Y)]_{c}$ for all $X, Y \in \mathcal{E}$, where $[\cdot, \cdot]_{c}$ is the commutator bracket on $\operatorname{Der}(\mathcal{A})$.

A Lie algebroid on a vector bundle $E$ over a base manifold $M$ is a Lie pseudoalgebra over the $\mathbb{R}$-algebra of smooth functions $\mathcal{A}=C^{\infty}(M)$ on the $C^{\infty}(M)$-module $\mathcal{E}=\operatorname{Sec}(E)$ of smooth sections of $E$. In this case the anchor map is described by a vector bundle morphism $a: E \rightarrow T M$ which induces the bracket homomorphism from $(\mathcal{E},[\cdot, \cdot])$ into the Lie algebra $\left(\mathcal{X}(M),[\cdot, \cdot]_{v f}\right)$ of vector fields on $M$.

Lie pseudoalgebras appeared first in the paper of Herz [He] but one can find similar concepts under more than a dozen of names in the literature (e.g. Lie modules, $(R, A)$-Lie algebras, Lie-Cartan pairs, Lie-Rinehart algebras, differential algebras, etc.). Lie algebroids were introduced by Pradines $[\mathrm{Pr}]$ as infinitesimal parts of differentiable groupoids. In the same year a book by Nelson was published where a general theory of Lie modules together with a big part of the corresponding differential calculus can be found. We also refer to a survey article by Mackenzie [Ma2].

\footnotetext{
*Supported by KBN, grant No 2 PO3A 04118
} 
Lie algebroids on a singleton base space are Lie algebras. Another canonical example is the tangent bundle $T M$ with the canonical bracket $[\cdot, \cdot]_{v f}$ on the space $\mathcal{X}(M)=\operatorname{Sec}(T M)$ of vector fields.

During Poisson seminars at the Warsaw University we realized long ago that the axiom (iii) telling that the anchor map induces a Lie algebra homomorphism is superfluous for Lie algebroids and that also the rest of assumptions can be weakened. The proofs are very elementary and we did not pay much attention to this result, especially when we discovered that such observations, although not explicitly in the language of Lie algebroids, had been already done in the old paper by Herz [He] and repeated by Kosmann-Schwarzbach and Magri ([KSM], section 6.1). However, to our surprise, all the time we find some people, even people working in the subject, who seem to have no knowledge about these simple facts, or rediscover them, or, in the worst case, try to build generalized object which are impossible due to these observations. The fact that these remarks concerning the axioms of a Lie algebroid seem not to be commonly known, encouraged us finally to publish the present note (circulating since a few years as a private communication) in which we discuss axioms of Lie algebroid and present a survey of relevant results in this direction. Our setting is slightly more general than just Lie algebroids, since we accept also certain brackets which do not satisfy the Jacobi identity (what is already present in [KSM]) or are not assumed to be skew-symmetric. We add also certain results on the axioms of Poisson and Jacobi brackets which can be of some interest in this context.

\section{Quasi-derivations in modules and vector bundles}

Let $\mathcal{A}$ be an associative ring (not necessarily commutative) over a commutative and unitary ring $R$ and let $\mathcal{E}$ be an $\mathcal{A}$-module. Our standard example will be the $C^{\infty}(M)$-module of sections of a vector bundle $E$ over $M$. We will write the operation in $\mathcal{A}$ and the $\mathcal{A}$-action on $\mathcal{E}$ without special signs: $\mathcal{A} \times \mathcal{A} \ni(f, g) \mapsto f g \in \mathcal{A}$ and $\mathcal{A} \times \mathcal{E} \ni(f, X) \mapsto f X \in \mathcal{E}$. For $f \in \mathcal{A}$ we denote by $f_{\mathcal{E}}$ the operator on $\mathcal{E}$ afforded by the module structure: $f_{\mathcal{E}}(X)=f X$. Let $\mathcal{A}_{\mathcal{E}}$ denote the set of all such operators. We call a linear operator $D: \mathcal{E} \rightarrow \mathcal{E}$ a quasi- $\mathcal{A}$-derivation if $\left[D, \mathcal{A}_{\mathcal{E}}\right]_{c} \subset \mathcal{A}_{\mathcal{E}}$, where the bracket operation $[\cdot, \cdot]_{c}$ is the commutator. Let $\operatorname{Qder}_{\mathcal{A}}(\mathcal{E})$ denote the $R$-module of all quasi- $\mathcal{A}$-derivations. We have the following (cf. [Sk], Proposition 1.1).

Theorem 1 If $\mathcal{E}$ is a faithful $\mathcal{A}$-module, then there is a linear mapping $\operatorname{Qder}_{\mathcal{A}}(\mathcal{E}) \ni D \mapsto \widehat{D} \in \operatorname{Der}(\mathcal{A})$ such that

$$
\left[D, f_{\mathcal{E}}\right]_{c}=(\widehat{D}(f))_{\mathcal{E}}
$$

Proof. By definition, for any $D \in \operatorname{Qder}_{\mathcal{A}}(\mathcal{E})$ there is $\widehat{D}(f) \in \mathcal{A}$ such that $\left[D, f_{\mathcal{E}}\right]_{c}=\widehat{D}(f)_{\mathcal{E}}$ and $\widehat{D}(f)$ is uniquely determined for any $f \in \mathcal{A}$, since the module is faithful. We have $D(f g X)=f g D(X)+$ $\widehat{D}(f g) X$. On the other hand,

$$
D(f g X)=f D(g X)+\widehat{D}(f) g X=f g D(X)+(f \widehat{D}(g)+\widehat{D}(f) g) X,
$$

so that $\widehat{D}(f g)=f \widehat{D}(g)+\widehat{D}(f) g$, since the module is faithful, i.e. $\widehat{D}$ is an $\mathcal{A}$-derivative. The mapping $D \mapsto \widehat{D}$ is clearly linear.

Let us assume for the future that we deal with faithful modules. The above map $\operatorname{Qder}_{\mathcal{A}}(\mathcal{E}) \ni D \mapsto$ $\widehat{D} \in \operatorname{Der}(\mathcal{A})$ we will call the universal anchor map for the $\mathcal{A}$-module $\mathcal{E}$. The universal anchor map induces the short exact sequence

$$
0 \rightarrow \operatorname{End}_{\mathcal{A}}(\mathcal{E}) \rightarrow \operatorname{Qder}_{\mathcal{A}}(\mathcal{E}) \rightarrow \operatorname{Der}(\mathcal{A}) .
$$

The following theorem shows that this is a short exact sequence of Lie algebras.

Theorem 2 The $R$-module $\operatorname{Qder}_{\mathcal{A}}(\mathcal{E})$ is closed with respect to the commutator. Moreover, the universal anchor map is a Lie algebra homomorphism, i.e.

$$
\left[\widehat{D_{1}, D_{2}}\right]_{c}=\left[\widehat{D_{1}}, \widehat{D_{2}}\right]_{c}
$$

for $D_{1}, D_{2} \in \operatorname{Qder}_{\mathcal{A}}(\mathcal{E})$. 
Proof. By definition,

$$
\begin{aligned}
{\left[D_{1}, D_{2}\right]_{c}(f X) } & =D_{1}\left(D_{2}(f X)\right)-D_{2}\left(D_{1}(f X)\right) \\
& =D_{1}\left(f D_{2}(X)+\widehat{D_{2}}(f) X\right)-D_{2}\left(f D_{1}(X)+\widehat{D_{1}}(f) X\right) \\
& =f\left(D_{1}\left(D_{2}(X)\right)-D_{2}\left(D_{1}(X)\right)+\widehat{D_{1}}\left(\widehat{D_{2}}(f)\right)-\widehat{D_{2}}\left(\widehat{D_{1}}(f)\right)\right) X \\
& =f\left[D_{1}, D_{2}\right]_{c}(X)+\left[\widehat{D_{1}}, \widehat{D_{2}}\right]_{c}(f) X .
\end{aligned}
$$

Hence, $\left[D_{1}, D_{2}\right]_{c}$ is a quasi-derivation and

$$
\left[\widehat{D_{1}, D_{2}}\right]_{c}(f) X=\left[\widehat{D_{1}}, \widehat{D_{2}}\right]_{c}(f) X
$$

for all $X \in \mathcal{E}$ and all $f \in \mathcal{A}$ and the theorem follows, since the module is faithful.

In case $\mathcal{A}$ is a commutative ring, $\operatorname{Qder}_{\mathcal{A}}(\mathcal{E})$ is additionally an $\mathcal{A}$-module with respect to the action $f D=f_{\mathcal{E}} \circ D$.

Proposition 1 If $\mathcal{A}$ is a commutative ring then the commutator bracket on the $\mathcal{A}$-module $\operatorname{Qder}_{\mathcal{A}}(\mathcal{E})$ satisfies

$$
\left[D_{1}, f D_{2}\right]_{c}=f\left[D_{1}, D_{2}\right]_{c}+\widehat{D_{1}}(f) D_{2} .
$$

Proof. It follows from the Leibniz rule for the commutator bracket:

$$
\left[D_{1}, f D_{2}\right]_{c}=\left[D_{1}, f_{\mathcal{E}} \circ D_{2}\right]_{c}=\left[D_{1}, f_{\mathcal{E}}\right]_{c} \circ D_{2}+f_{\mathcal{E}} \circ\left[D_{1}, D_{2}\right]_{c}
$$

Corollary 1 If $\mathcal{A}$ is commutative then $\operatorname{Qder}_{\mathcal{A}}(\mathcal{E})$ is canonically a Lie pseudoalgebra over $R$ and $\mathcal{A}$.

It is pointed out in [KSMk] that concept of quasi-derivation can be traced back to N. Jacobson [Ja1, Ja2] as a special case of his pseudo-linear endomorphism. It has appeared also in [Ne] under the name of a module derivation and used to define linear connections in the algebraic setting. In the geometric setting for Lie algebroids it has been studied in [Ma1], Ch. III, under the name covariant differential operator. For more detailed history and recent development we refer to [KSMk] where quasi-derivations are called derivative endomorphisms.

\section{QD-algebroids}

Suppose now that $\mathcal{E}$ is faithful and that on $\mathcal{E}$ we have additionally a bracket operation $[\cdot, \cdot]$ which is $R$-linear in the second argument and satisfies the Jacobi identity (1), i.e. $\operatorname{ad}_{[X, Y]}=\left[\operatorname{ad}_{X}, \operatorname{ad}_{Y}\right]_{c}$, where $\operatorname{ad}_{X}(Y)=[X, Y]$ is the adjoint operator. Let us assume that the adjoint operators ad $\operatorname{ad}_{X}$ are in $\operatorname{Qder}_{\mathcal{A}}(\mathcal{E})$ for any $X \in \mathcal{E}$. We can define the anchor map $\mathcal{E} \ni X \mapsto \widehat{X} \in \operatorname{Der}(\mathcal{A})$ by $\widehat{X}=\widehat{\operatorname{ad}_{X}}$.

Proposition 2 The anchor map is a bracket homomorphism, i.e.

$$
\widehat{[X, Y]}=[\widehat{X}, \widehat{Y}]_{c}
$$

for all $X, Y \in \mathcal{E}$.

Proof. This is a direct consequence of Theorem 2 and the Jacobi identity.

Consider a particular example, where $\mathcal{A}$ is the commutative associative algebra of smooth functions on a manifold $M$ and $\mathcal{E}$ is the $\mathcal{A}$-module of smooth sections of a vector bundle $E$ over $M$ with $k$ dimensional fibers. Note that this module is faithful. In this case quasi-derivations are particular first-order differential operators on $E$. An $\mathbb{R}$-bilinear bracket $[\cdot, \cdot]$ on $\mathcal{E}$ we will call a quasi-derivation algebroid (shortly $Q D$-algebroid) if the bracket is a quasi- $\mathcal{A}$-derivation of $\mathcal{E}$ for both variables separately. 
Hence, $[X, f Y]=f[X, Y]+\widehat{X}(f) Y$ and $[f X, Y]=f[X, Y]+\tilde{Y}(f) X$ for all $X, Y \in \mathcal{E}$ and all $f \in \mathcal{A}$, where $X \mapsto \widehat{X} \in \operatorname{Der}(\mathcal{A})$ and $Y \mapsto \widetilde{Y} \in \operatorname{Der}(\mathcal{A})$ are, respectively, the left and the right anchor maps. In the case when the anchor maps are tensorial ( $\mathcal{A}$-linear), we speak about an algebroid (cf. [GU]).

In the case when the bracket is a Lie algebra bracket, we will speak about a Lie QD-algebroid (resp., Lie algebroid). We can also consider Loday QD-algebroid (resp., Loday algebroid) requiring additionally only the Jacobi identity (1) without skew-symmetry assumption. The name is motivated by Loday algebras, i.e. the concept of 'non-skew-symmetric Lie algebras' introduced by J. L. Loday [Lo1, Lo2] (and called by Loday Leibniz algebras).

Theorem 3 Every QD-algebroid (resp., Loday QD-algebroid, or Lie QD-algebroid) of rank $>1$ is an algebroid (resp., Loday algebroid, or Lie algebroid).

Proof. According to Theorem 2, we have $[X, f Y]=f[X, Y]+\widehat{X}(f) Y$ and $[f X, Y]=f[X, Y]+\widetilde{Y}(f) X$ for all $X, Y \in \mathcal{E}$ and all $f \in \mathcal{A}$, where $\widehat{X}$ and $\widetilde{Y}$ are derivations of $\mathcal{A}$. Moreover, due to Proposition 2, in the case of a Loday QD-algebroid we have $\widehat{[X, Y]}=[\widehat{X}, \widehat{Y}]_{c}$. For a Lie QD-algebroid, additionally, $\widehat{X}=-\tilde{X}$. It remains to show that for QD-algebroids of rank $>1$ the maps $X \mapsto \widehat{X}$ and $Y \mapsto \widetilde{Y}$ are $\mathcal{A}$-linear. To see this, let us write $[g X, f Y]$ in two different ways. First,

$$
[g X, f Y]=f[g X, Y]+\widehat{g X}(f) Y=f g[X, Y]+f \widetilde{Y}(g) X+\widehat{g X}(f) Y .
$$

On the other hand,

$$
[g X, f Y]=g[X, f Y]+\widetilde{f Y}(g) X=g f[X, Y]+g \widehat{X}(f) Y+\widetilde{f Y}(g) X
$$

so that

$$
f \widetilde{Y}(g) X+\widehat{g X}(f) Y=g \widehat{X}(f) Y+\widetilde{f Y}(g) X
$$

or

$$
(\widehat{g X}-g \widehat{X})(f) Y=(\widetilde{f Y}-f \widetilde{Y})(g) X .
$$

But, since the rank of the bundle is $>1$, for a given $X \in \mathcal{E}$ we can choose $Y$ which is locally linearly independent (and vice versa), so that (3) implies $\widehat{g X}-g \widehat{X}=0$ and $\widetilde{f Y}-f \widetilde{Y}=0$ for any $X, Y \in \mathcal{E}$ and $f \in \mathcal{A}$.

As it has been proved in [GM1], the difference between Loday algebroids and Lie algebroids is not very big.

Theorem 4 For any Loday algebroid, the left and the right anchor differ by sign, $\widetilde{X}=-\widehat{X}$, and the bracket is skew-symmetric at points of $M$ where they do not vanish.

In other words, a Loday algebroid is, in fact a Lie algebroid around points where the anchors (or, equivalently, one of the anchors) do not vanish, and it has Loday algebras as fibers over points where the anchors do vanish.

Consider now QD-algebroids of rank 1. The bracket defined on sections of a line-bundle $E$ over $M$ is a differential operator of first order, so local. Assuming the Jacobi identity for the bracket, i.e. dealing with Loday QD-algebroid, one can prove, in fact, skew-symmetry of the bracket ([GM2], Corollary 2).

Theorem 5 Every Loday QD-algebroid of rank 1 is a Lie QD-algebroid.

A Lie QD-algebroid of rank 1 is just a local Lie algebra in the sense of Kirillov [Ki]. In the trivial case $E=M \times \mathbb{R}$ the bracket is defined on $C^{\infty}(M)=S e c(E)$ and it is called a Jacobi bracket [Li]. It is of the form

$$
[f, g]=\Lambda(\mathrm{d} f, \mathrm{~d} g)+f \Gamma(g)-g \Gamma(f)
$$


for certain bivector field $\Lambda$ and certain vector field $\Gamma$. The Jacobi identity is equivalent to compatibility conditions, usually written in terms of the Schouten-Nijenhuis bracket:

$$
[\Gamma, \Lambda]^{S N}=0, \quad[\Lambda, \Lambda]^{S N}+2 \Lambda \wedge \Gamma=0 .
$$

One has $\widehat{f}=i_{\mathrm{d} f} \Lambda+f \Gamma$. In the case when $\Gamma=0$ we deal with a Poisson bracket and in the case $\Lambda=0$ - with a Lie algebroid bracket. This means that Lie algebroids on the trivial 1-dimensional bundle (i.e. Lie algebroid brackets on $\left.C^{\infty}(M)\right)$ are of the form $[f, g]=f \Gamma(g)-g \Gamma(f)$ for certain vector field $\Gamma$ on $M$. Here the anchor map is $\widehat{f}=f \Gamma$. Other natural examples of rank-1 Lie algebroids are associated with foliations of $M$ by one-dimensional leaves.

Of course, Theorem 5 implies in particular that we can skip skew-symmetry in the definition of Jacobi and Poisson brackets. The direct proof for Poisson brackets is particularly simple. Indeed, the Jacobi identity (1) implies immediately that $[[f, g]+[g, f], h]=0$ for all $f, g, h \in C^{\infty}(M)$. Using the Leibniz rule with respect to both arguments, we get

$$
0=\left[\left[f^{2}, g\right]+\left[g, f^{2}\right], h\right]=2([f, g]+[g, f])[f, h] .
$$

For $f=g=h$ we get $4[f, f]^{3}=0$, thus $[f, f]=0$ and skew-symmetry follows.

Let us summarize the above results as follows.

Theorem 6 Let $E$ be a vector bundle over $M$ and let $[\cdot, \cdot]$ be a bilinear bracket operation on the $C^{\infty}(M)$-module $\mathcal{E}=\operatorname{Sec}(E)$ that satisfies the Jacobi identity (1) and that is a quasi-derivation with respect to both arguments.

(a) If $\operatorname{rank}(E)>1$, then there is a vector bundle morphism a: $E \rightarrow T M$ over the identity map on $M$ such that $a([X, Y])=[a(X), a(Y)]_{v f}$ and

$$
[f X, g Y]=f g[X, Y]+f a(X)(g) Y-g a(Y)(f) X
$$

for all $X, Y \in \mathcal{E}, f, g \in C^{\infty}(M)$. Moreover, $[X, Y](p)=-[Y, X](p)$ if $a_{p} \neq 0$.

(b) If $\operatorname{rank}(E)=1$, then the bracket is skew-symmetric and defines a local Lie algebra structure which, locally, is equivalent to a Jacobi bracket (4).

Corollary 2 Lie $Q D$-algebroids on $E$ are exactly Lie algebroids if $\operatorname{rank}(E)>1$, and local Lie algebras in the sense of Kirillov if $\operatorname{rank}(E)=1$.

Corollary 3 A Lie algebroid on a vector bundle $E$ of rank $>1$ is just a Lie bracket on sections of $E$ which is a quasi-derivation with respect to one (hence both) argument.

Acknowledgments. The author wish to thank the referees for useful comments.

\section{References}

[GM1] Grabowski, J. and Marmo, G., Non-antisymmetric versions of Nambu-Poisson and Lie algebroid brackets, J. Phys. A: Math. Gen. 34 (2001), 3803-3809.

[GM2] Grabowski, J. and Marmo, G., Binary operations in classical and quantum mechanics, to appear in "Classical and Quantum Integrability", Banach Center Publications, vol. 59.

[GU] Grabowski, J. and Urbański, P., Algebroids - general differential calculi on vector bundles, J. Geom. Phys. 31 (1999), 111-141.

[He] Herz, J. C., Pseudo-algèbres de Lie, C. R. Acad. Sci. Paris 236 (1953), I, pp. 1935-1937, II, pp. 2289-2291. 
[Ja1] Jacobson, N., On pseudo-linear transformations, Proc. Nat. Acad. Sci. 21 (1935), 667-670.

[Ja2] Jacobson, N., Pseudo-linear transformations, Ann. Math. 38 (1937), 485-506.

[Ki] Kirillov, A. A., Local Lie algebras (Russian), Uspekhi Mat. Nauk 31 (1976), 57-76.

[KSMk] Kosmann-Schwarzbach, Y. and Mackenzie, K., Differential operators and actions of Lie algebroids, in "Quantization, Poisson brackets and beyond", ed. T. Voronov, Contemporary Math. 315 (2002), 213-233.

[KSMg] Kosmann-Schwarzbach, Y. and Magri F., Poisson-Nijenhuis structures, Ann. Inst. Henri Poincaré A53 (1990), 35-81.

[Li] Lichnerowicz, A.: Les variétés de Jacobi et leurs algébres de Lie associées, J. Math. Pures Appl., 57 (1978), 453-488.

[Ne] Nelson, E.: Tensor Analysis, Princeton University Press and The University of Tokyo Press, Princeton 1967.

[Lo1] Loday, J.-L., Cyclic Homology, Springer Verlag, Berlin 1992.

[Lo2] Loday, J.-L., Une version non commutative des algèbres de Lie: les algèbres de Leibniz, Ann. Inst. Fourier 37 (1993), 269-93.

[Ma1] Mackenzie, K.: Lie Groupoids and Lie Algebroids in Differential Geometry, Cambridge University Press, 1987.

[Ma2] Mackenzie, K., Lie algebroids and Lie pseudoalgebras, Bull. London Math. Soc. 27 (1995), $97-147$.

[Pr] Pradines, J., Théorie de Lie pour les groupoïdes différentiables. Calcul différentiel dans la catégorie des groupoïdes infinitésimaux, C. R. Acad. Sci. Paris, Sér. A, 264 (1967), 245-248.

[Sk] Skryabin, S. M., An algebraic approach to the Lie algebras of Cartan type, Comm. Algebra 21 (1993), 1229-1336. 